\title{
X-raying Circumstellar Material around Young Stars
}

\author{
P. C. Schneider ${ }^{1}$ and H. M. Günther ${ }^{2}$ \\ ${ }^{1} \mathrm{ESA} / \mathrm{ESTEC}$ \\ email: cschneid@cosmos.esa.int \\ ${ }^{2} \mathrm{MIT}$ \\ email: hgunther@mit.edu
}

\begin{abstract}
Young stars are surrounded by copious amounts of circumstellar material. Its composition, in particular its gas-to-dust ratio, is an important parameter. However, measuring this ratio is challenging, because gas mass estimates are often model dependent. X-ray absorption is sensitive to the gas along the line-of-sight while optical/near-IR extinction depends on the dust. Therefore, the absorber's gas-to-dust ratio is directly given by the ratio between X-ray and optical/near-IR extinction. We present three systems where we used X-ray and optical/near-IR data to constrain the gas-to-dust ratio of circumstellar material; from a dust rich debris disk to gaseous protoplanetary disks.
\end{abstract}

\section{Introduction}

The chemical composition of circumstellar disks is one of their most important properties. It controls many transport phenomena within the disk like accretion, angular momentum transport as well as planet formation. The interstellar medium (ISM) consists mainly of gas; only a small fraction $(\sim 1 \%)$ is in the form of dust grains. Protoplanetary disks are thought to have a gas-to-dust ratio close to the ISM value during their early evolutionary stages. Therefore, the ISM gas-to-dust ratio of 100:1 is often assmued when deriving the total disk mass from dust measurements. Directly deriving the total gas reservoir is more challenging as the conversion of gas emission line fluxes to total (gas) disk mass strongly depends on often uncertain model parameters.

One possibility to trace the gas is X-ray absorption. The absorption cross section is essentially independent of the chemical bonds of the absorbing material, because X-ray absorption involves the inner shell electrons while molecular bonds involve the outer (valence) electrons. Thus, we can derive the absorber's gas content from its X-ray absorption signal. Comparison with optical extinction caused by the dust along the line-of-sight enables us to measure the gas-to-dust ratio of the absorbing material.

The location of the absorber(s) along the line-of-sight often remains uncertain. Time variability of the absorption can help to overcome this ambiguity. In this proceedings, we present three cases where the location of the absorption can be determined with some certainty so that a local gas-to-dust ratio can be derived.

\section{The debris disk around AU Mic}

AU Mic is a young (23 \pm 3 Myrs, e.g. Mamajek et al. 2015) M1 dwarf at a distance of about $10 \mathrm{pc}$ belonging to the $\beta$ Pic moving group (Zuckerman et al. 2001). AU Mic is surrounded by an edge-on debris disk, i.e., a dust rich disk. This disk, extending out to $\sim 150 \mathrm{AU}$, has been subject to numerous studies from scattered light (e.g. Kalas et al. 2004, Krist et al. 2005), over dust emission (Liu et al. 2004) to absorption (Roberge et al. 

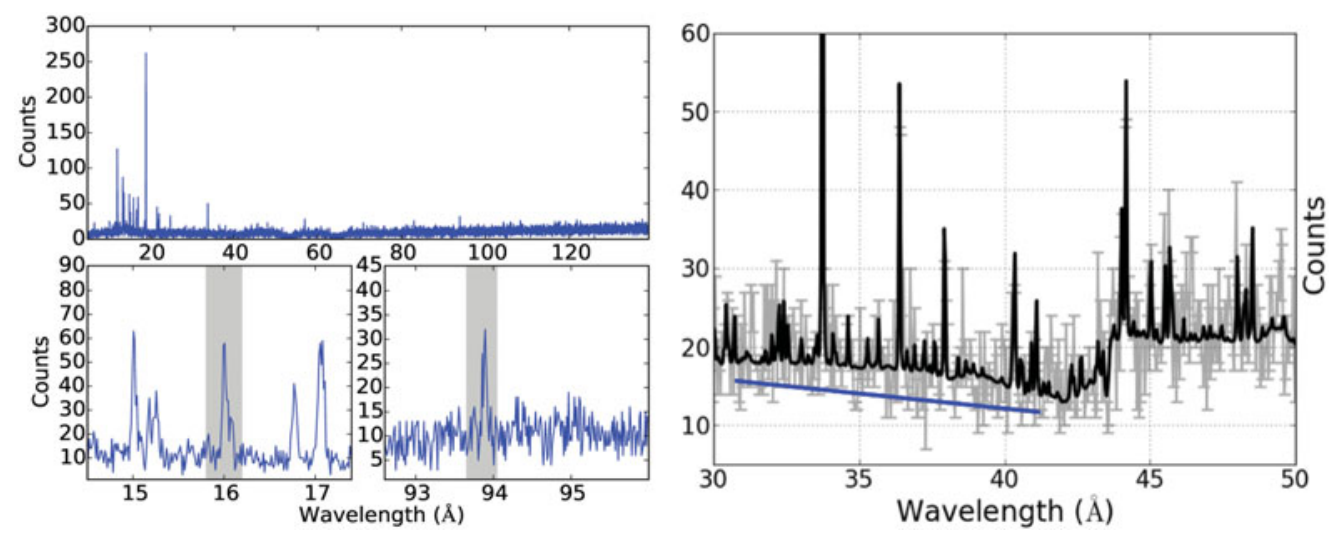

Figure 1. Left: Chandra LETGS spectrum of AU Mic. As an example, two Fe XVIII lines that were used to derive the absorbing column density are highlighted. Right: The carbon K-edge. The edge at $43 \AA$ is clearly visibile, but can be entirely explained by carbon within the filter in front of the X-ray detector. Additional carbon within the AU Mic disk would lower the flux shortwards of $43 \AA$ as indicated by the blue line.

2005) and molecular hydrogen emission (France et al. 2007). Given its small distance to the Earth, we can exclude substantial interstellar absorption so that any absorption signal can be safely associated with AU Mic's circumstellar disk.

Thanks to its youth and distance, AU Mic is a bright X-ray source on the sky (e.g. Linsky et al. 2002). We obtained a Chandra LETGS X-ray spectrum of AU Mic (see Fig. 1, published in Schneider \& Schmitt 2012), which extends from a few $\AA$ up to $>100 \AA$. This wavelengths coverage allowed us to compare lines of the same element and ionization stage formed at largely different wavelengths. Their line ratios are given mainly by atomic physics and almost independent of the plasma temperature. Thus, these line ratios can be used to measure of the total absorption towards AU Mic. Specifically from our analysis of abundant Fe lines, we were able to limit the gas column density to $N_{H}<10^{19} \mathrm{~cm}^{-2}$ (1 $\sigma$ conf.). Furthermore, this spectrum allowed us to directly constrain the amount of carbon in gas and small grains $(\lesssim 0.5 \mu \mathrm{m})$ from the depth of the carbon K-edge. Figure 1 (right) shows a zoom into the wavelengths region around the C-K edge. The edge-like structure is clearly visible. However, its depth can be entirely explained by carbon in the detector itself. We derive an upper limit on the carbon content of $N_{C}<10^{18} \mathrm{~cm}^{-2}$.

Comparing our upper limits with published dust masses, we find that the disk must contain more dust than gas. The measurements of Fitzgerald et al. 2007 suggest a dust mass around $0.01 M_{\oplus}$ (France et al. 2007), which would correspond to a total dust column density of $7 \times 10^{-4} \mathrm{~g} \mathrm{~cm}^{-2}$ or $3 \times 10^{20} \mathrm{~cm}^{-2}$ for ISM abundances. That we detect less absorption implies that the dust is locked in large grains that are opaque to X-rays.

\section{The protoplanetary disk around the CTTS AA Tau}

The classical T Tauri star (CTTS) AA Tau is surrounded by a gaseous protoplanetary disk. Compared to AU Mic, AA Tau is younger ( 2 Myrs) and its disk is not viewed edge-on, but inclined by $75^{\circ}$ with respect to the plane of the sky. Thus, the disk is not in front of the star for most of the time. The inner disk region, however, is warped and periodically eclipses the star. X-ray data showed that this inner region is gas-rich as measured by $N_{H} / A_{V}$ where $N_{H}$ is the equivalent hydrogen column density derived from 

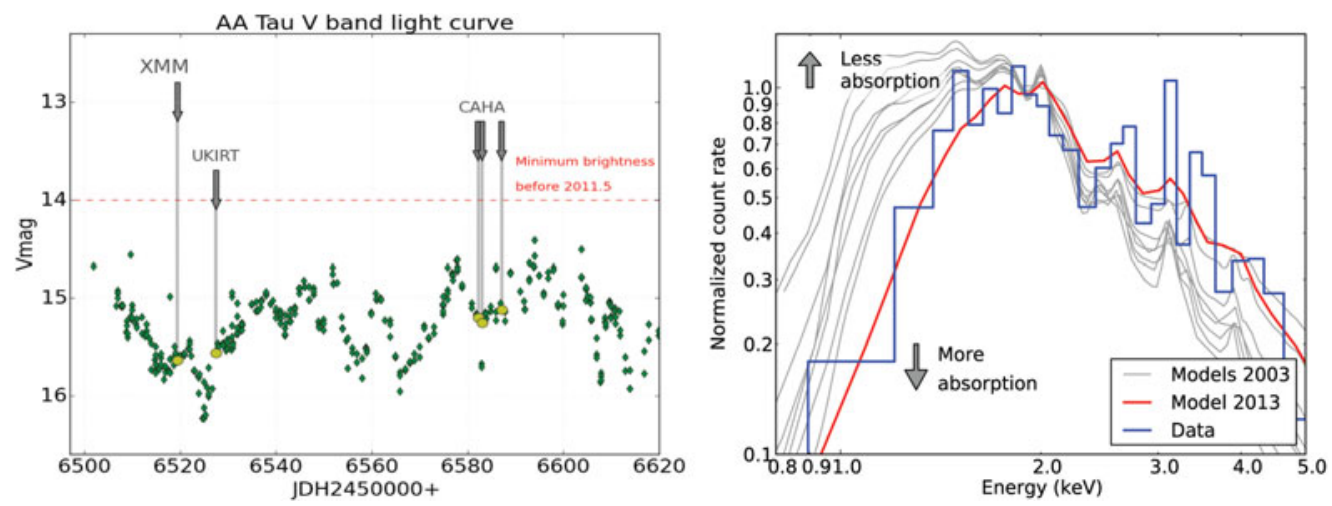

Figure 2. Left: Optical light curve of AA Tau around the X-ray and near-IR observations. Right: XMM-Newton pn spectrum of AA Tau with models normalized to the count rate at $E_{\text {phot }}=2.0 \mathrm{keV}$.

low-resolution X-ray spectra and $A_{V}$ is the dust extinction (Schmitt \& Robrade 2007, Grosso et al. 2007).

End of 2011, the system darkened significantly in the optical $(\Delta V \approx 2$, see Fig. 2 left). Bouvier et al. (2013) suggest that the extra absorption is caused by disk material at larger radii ( $r \gtrsim 10 \mathrm{AU})$, which rotated into view and now obscures the line-of-sight towards the central star. That the inner disk region is obscured from view is confirmed by our high-resolution HST FUV spectrum obtained during the dim state, as the $\mathrm{H}_{2}$ line width decreased compared to the bright state's data published by France et al. 2012. We obtained a new X-ray observation with XMM-Newton during the dim state. It reveals that the increase in absorbing column density is moderate $\left(N_{H}^{e x t r a}=0.5-1.0 \times 10^{22} \mathrm{~cm}^{-2}\right)$. Our contemporaneous UV to near-IR data characterize the dust extinction during the dim state. Comparing dust and gas absorption, we find a gas-to-dust ratio of $N_{H} / A_{V}=$ $0.8 \ldots 3.6 \times 10^{21}$, which is within a factor of two of the ISM value (Schneider et al., A\&A submitted).

\section{RW Aur: Another CTTS during a dim state}

RW Aur consists of two CTTSs; both of approximately solar mass. The system underwent a major dimming event towards the end of 2014 ( $\Delta V=2$ to 3 magnitudes). This event resembles a previously observed half-year long dimming, which was attributed to a tidal stream between both components (Rodriguez et al. 2013, Dai et al. 2015). However, this new dimming event might more closely resemble the AA Tau dimming with obscuration by the primary's disk itself as suggested by new infrared data (see Shenavrin et al. 2015).

We obtained a new Chandra observation of the system during a dim state that started end of 2014 (see Fig. 3). Comparing the dim state's X-ray properties with those observed previously by Skinner \& Güdel (2014) during the bright state, we find an increase in absorbing column density of $N_{H} \approx 2 \times 10^{22} \mathrm{~cm}^{-2}$. Antipin et al. (2015) find that the optical extinction appears rather gray, i.e., that the absorption is caused by grains with sizes greater than $1 \mu \mathrm{m}$. Therefore, the dust mass might differ from the value suggested by assuming $\Delta V=A_{V}$ and an ISM-like grain population. As an estimate, we assume absorption by $1 \mu \mathrm{m}$ sized grains and opacities from Draine (2003). With these assumptions, the gas-to-dust ratio of the extra absorber is below the ISM-value when comparing 

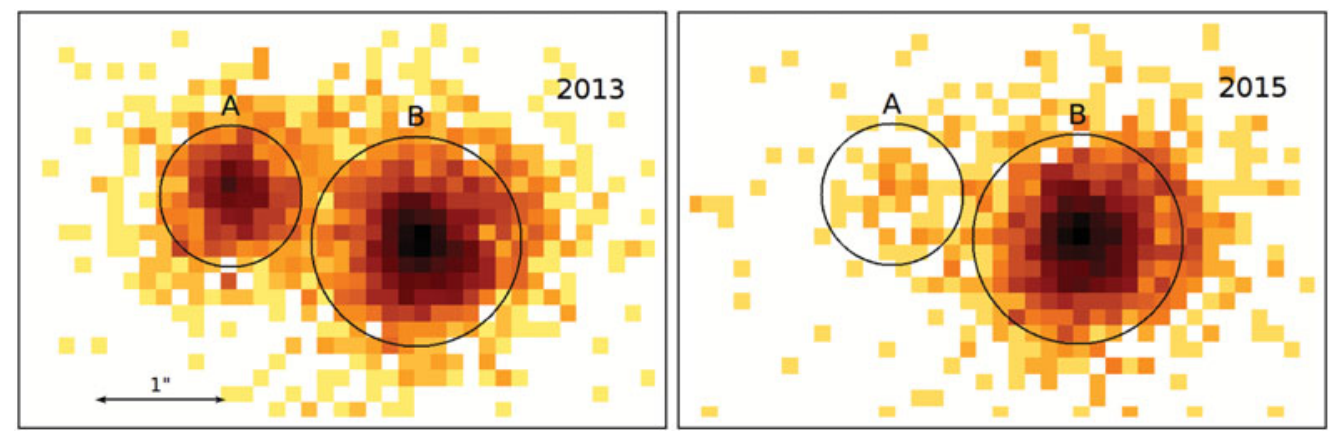

Figure 3. Chandra images of the RW Aur system. Left: Bright state, Right: dim state with the tidal stream in front of the A component.

the observed 2 mag of dust extinction with the increase in X-ray absorption (Schneider et al., in prep.).

\section{Conclusions}

The gas-to-dust ratio of circumstellar material is probed by the ratio between X-ray absorption $\left(N_{H}\right)$ and optical/NIR extinction $\left(A_{V}\right)$. We present three systems where we applied this method. The debris disk system AU Mic contains more grains than gas, the protoplanetary disk around the CTTS AA Tau has a gas-to-dust ratio compatible with the ISM at radii of a few AU, and the absorber in the young binary system RW Aur has low gas-to-dust ratio and large grains.

\section{References}

Antipin, S., Belinski, A., Cherepashchuk, A., Cherjasov, D., Dodin, A., Gorbunov, I., Lamzin, S., Kornilov, M., Kornilov, V., Potanin, S., Safonov, B., Senik, V., Shatsky, N., \& Voziakova, O. $2015 I B V S, 6126,1$

Bouvier, J., Grankin, K., Ellerbroek, L. E., Bouy, H., \& Barrado, D., 2013, A\& A, 557, A77

Dai, F., Facchini, S., Clarke, C. J., \& Haworth, T. J. 2015 MNRAS, 449, 1996

Draine, B. T. 2003, ARAA, 41, 241

Fitzgerald, M. P., Kalas, P. G., Duchêne, G., Pinte, C. \& Graham, J. R., 2007 ApJ, 670, 536F

France, K., Roberge, A., Lupu, R. E., Redfield, S., \& Feldman, P. D., 2007 ApJ, 668, 1174

France, K., Burgh, E. B., Herczeg, G. J., Schindhelm, E. Yang, H., Abgrall, H., Roueff, E., Brown, A., Brown, J. M., \& Linsky, J. L., 2012 ApJ, 744, 22F

Grosso, N., Bouvier, J., Montmerle, T., Fernández, M., Grankin, K., \& Zapatero Osorio, M. R., 2007 A 6 A, 475, 607

Kalas, P., Liu, M. C., \& Matthews, B. C. 2004, Science, 303, 1990

Krist, J. E., Ardila, D. R., Golimowski, D. A., Clampin, M., et al. 2005, AJ, 129, 1008

Linsky, J. L., Ayres, T. R., Brown, A., \& Osten, R. A., 2002 Astron. Nachr., 323, 321

Liu, M. C., Matthews, B. C., Williams, J. P., \& Kalas, P. G., 2004 ApJ, 608, 526

Mamajek, E. E. \& Bell, C. P. M. 2015, MNRAS, 445, 2169

Roberge, A., Weinberger, A. J., Redfield, S., \& Feldman, P. D., 2005 ApJL, 626, 105

Rodriguez, J. E., Pepper, J., Stassun, K. G., Siverd, R. J., Cargile, P., Beatty, T. G., \& Gaudi, B. S, 2013 AJ, 146, 112

Shenavrin, V. I., Petrov, P. P., \& Grankin, K. N., 2015 IBVS, 6143, 1S

Schmitt, J. H. M. M. \& Robrade, J., 2007 AEAA, 462, 41

Schneider, P. C. \& Schmitt, J. H. M. M., $2012 A \& A, 516,8$

Skinner, S. L. \& Güdel, M. 2014 ApJ, 788, 101

Zuckerman, B., Song , I., Bessell, M. S., \& Webb, R. A. 2001, ApJL, 562, L87 